\title{
Exact boundary conditions in numerical relativity using multiple grids: scalar field tests
}

\author{
Gioel Calabrese \\ School of Mathematics, University of Southampton, Southampton, SO17 1BJ, \\ UK
}

\begin{abstract}
.
Cauchy-Characteristic Matching (CCM), the combination of a central $3+1$ Cauchy code with an exterior characteristic code connected across a timelike interface, is a promising technique for the generation and extraction of gravitational waves. While it provides a tool for the exact specification of boundary conditions for the Cauchy evolution, it also allows to follow gravitational radiation all the way to infinity, where it is unambiguously defined.

We present a new fourth order accurate finite difference CCM scheme for a first order reduction of the wave equation around a Schwarzschild black hole in axisymmetry. The matching at the interface between the Cauchy and the characteristic regions is done by transfering appropriate characteristic/null variables. Numerical experiments indicate that the algorithm is fourth order convergent. As an application we reproduce the expected late-time tail decay for the scalar field.

PACS numbers: 02.60.--x, 02.70.-c, 04.20.-q, 0425.Dm
\end{abstract}

\section{Introduction}

In the past year there has been remarkable progress in the field of numerical relativity, particularly in the simulation of binary black hole systems. Some of today's codes are capable of tracking the holes for many orbits before the merger and ringdown [1, 2, 3]. Since a primary goal of numerical relativity is the production of gravitational wave templates associated with the merger of black holes, it is important to simultaneously make progress with the tools which allow for their accurate generation and extraction.

The schemes which have been able to push forward the binary black hole problem either use spatial compactification [1, which brings spatial infinity at a finite coordinate location, or mesh refinement 2, 3, which allows the boundary to be placed further out by using lower resolution far away from the strong field region. A limitation of both methods is that the number of grid-points per wavelength gradually diminishes as the radiation propagates away from the sources and therefore the quality of the gravitational radiation progressively deteriorates, forcing the extraction to occur at a relatively small distance from the final holeł. The undesirable effect of a time-like

$\ddagger$ Another reason for extracting radiation at small distances is to avoid boundary effects. Nevertheless, the comparison of the real part of $r \psi_{4}$ extracted at $r=20 M$ and $r=40 M$ done by Baker et al. 2 seems to indicate that the extraction at small distances leads to sufficiently accurate results. 
boundary with a chronological future that intersects the region of interest (where physical quantities are calculated), was highlighted in [4, 5].

To improve the quality of the outgoing radiation one should aim to keep the coordinate distance that the waves have to travel to a minimum, without making their speed go to zero. This can be achieved, for example, by employing asymptotically null slices [6, 7, 8, 9, 10], or by supplementing the Cauchy evolution with a characteristic code 11, 12, 13, 14, 15, 16, 17, 18, 19, 20, 21, 22, 23, 24, 25, 26, which extends all the way to infinity. See Figures 12 and 3 Both methods resolve incoming waves poorly at very large distances, but these are not expected to be significant. The reason for not using a single characteristic evolution scheme, without a Cauchy interior covering the strong field region, is that such a scheme tends to develop caustics, where neighbouring characteristics focus and the coordinate system breaks down.

In this work we describe a fourth order accurate algorithm for the axisymmetric massless Klein-Gordon scalar field around a Schwarzschild black hole, which employs a Cauchy evolution scheme, based on space-like slices, and a characteristic scheme, based on null slices. The outer boundary of the interior Cauchy region is a timelike surface and coincides with the inner boundary of the characteristic region. Communication between the two regions is done taking into account the propagation of the characteristic variables. At the discrete level the interface is implemented with touching grids. We find that when the slices are not simultaneous, this particular multi-grid approach is better suited than overlapping grids, as it avoids complications due to causality. The radial coordinate of the characteristic region is compactified in order to reach future null infinity.

The paper is organised as follows. In Section 2 we describe the continuum scalar field initial-boundary value problem in the Cauchy and characteristic regions and discuss the interface treatment. In Section 3 we provide details of the discretization method and in Section 4 we describe the tests carried out to validate the code, including the simulation of the late-time tail decay.

\section{Klein-Gordon scalar field around a Schwarzschild black hole}

In this section we describe the continuum problem, giving the explicit form of the equations, and the interface treatment. The Schwarzschild line element in Kerr-Schild coordinates takes the form

$$
d s^{2}=-d t^{2}+d r^{2}+\frac{2 M}{r}(d t+d r)^{2}+r^{2} d \Omega^{2},
$$

where $M$ is the mass of the black hole and $d \Omega^{2}=d \theta^{2}+\sin \theta^{2} d \phi^{2}$. The surface defined by

$$
t=r+4 M \ln (r-2 M)+\text { const }
$$

is null and its normal, provided it belongs to the future null cone, points toward increasing values of $r$. It is convenient to introduce the shorthand $r^{ \pm}=r \pm 2 M$. Using $\Psi=r \Phi$, in the Cauchy region the wave equation on the Schwarzschild background, $\nabla^{\mu} \nabla_{\mu} \Phi=0$, takes the form

$\partial_{t}^{2} \Psi=\frac{4 M}{r^{+}} \partial_{r} \partial_{t} \Psi+\frac{r^{-}}{r^{+}} \partial_{r}^{2} \Psi-\frac{2 M}{r r^{+}} \partial_{t} \Psi+\frac{2 M}{r r^{+}} \partial_{r} \Psi-\frac{2 M}{r^{+} r^{2}} \Psi+\frac{\partial_{\theta}\left(\sin \theta \partial_{\theta} \Psi\right)}{r r^{+} \sin \theta}$.

In Bondi coordinates the Schwarzschild line element is

$$
d s^{2}=\left(-1+\frac{2 M}{r}\right) d u^{2}-2 d u d r+r^{2} d \Omega^{2}
$$




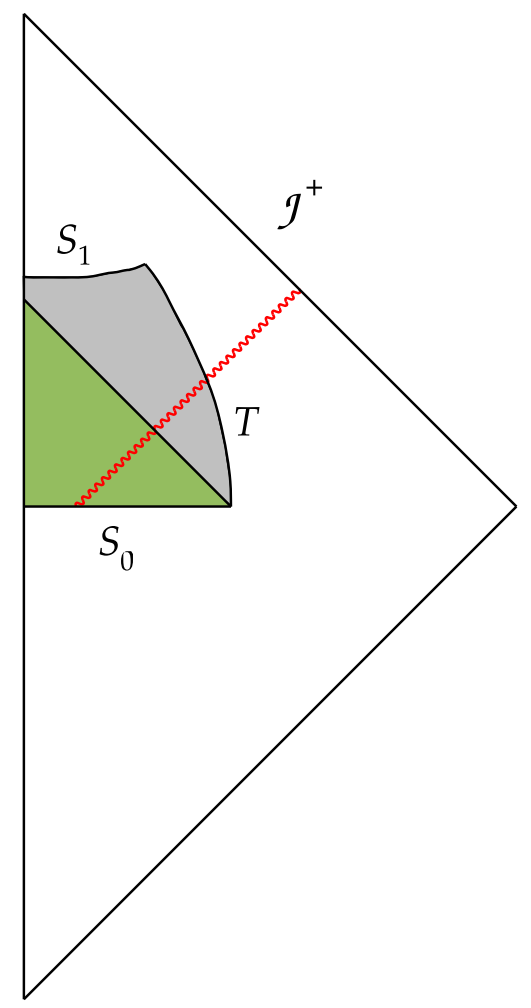

Figure 1. Most simulations in numerical relativity are carried out on a domain bounded by two space-like slices, $S_{0}$ and $S_{1}$ (the initial and final time), and a timelike artificial boundary $T$. Since it is not known how to specify local boundary conditions which do not introduce spurious radiation or reflect outgoing waves, the accuracy of the solution is limited to the future domain of dependence of $S_{0}$ (green region). The solution in the grey region, which is causally connected to the boundary, is inaccurate. In this situation one is forced to extract gravitational radiation relatively close to the sources.

and it is related to (11) via the coordinate transformation

$$
\begin{aligned}
& u=t-r-4 M \ln (r-2 M), \\
& \tilde{r}=r, \\
& \tilde{\theta}=\theta, \\
& \tilde{\phi}=\phi,
\end{aligned}
$$

which implies

$$
\partial_{t}=\partial_{u}
$$




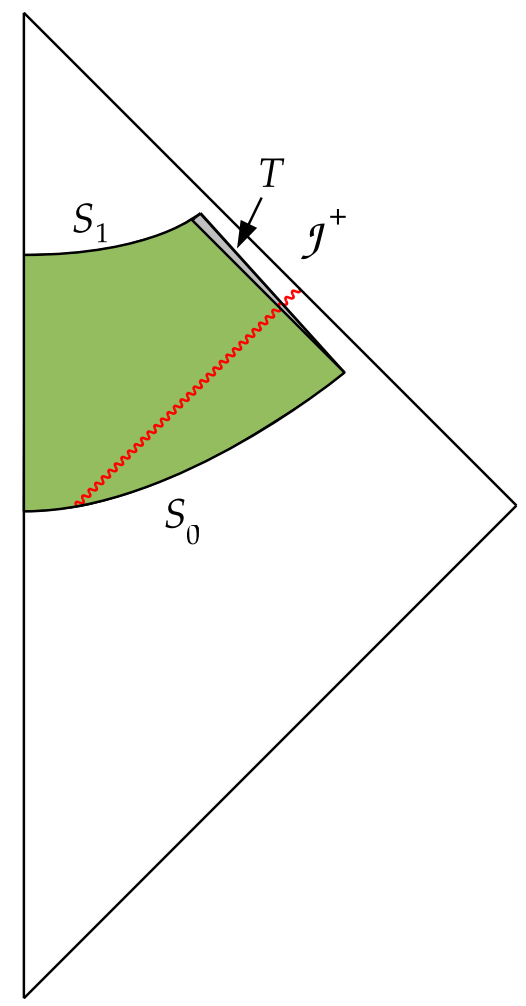

Figure 2. When asymptotically null slices are used, as in 27, the outer boundary $T$ can be placed far away from the sources and is approximately null (the incoming modes travel very slowly). Gravitational radiation can be accurately extracted at much larger distances and with lower computational cost than with standard space-like slices. See Table I of 27] for a comparison of computational costs and errors with different slices.

$$
\begin{aligned}
\partial_{r} & =-\frac{\tilde{r}+2 M}{\tilde{r}-2 M} \partial_{u}+\partial_{\tilde{r}}, \\
\partial_{\theta} & =\partial_{\tilde{\theta}}, \\
\partial_{\phi} & =\partial_{\tilde{\phi}} .
\end{aligned}
$$

Where there is no cause for confusion we will not distinguish between $\{\tilde{r}, \tilde{\theta}, \tilde{\phi}\}$ and $\{r, \theta, \phi\}$.

The Klein-Gordon equation in the characteristic region becomes

$$
0=-\frac{2 r}{r^{+}} \partial_{u} \partial_{\tilde{r}} \Psi+\frac{r^{-}}{r^{+}} \partial_{\tilde{r}}^{2} \Psi+\frac{2 M}{r r^{+}} \partial_{\tilde{r}} \Psi-\frac{2 M}{r^{+} r^{2}} \Psi+\frac{\partial_{\tilde{\theta}}\left(\sin \theta \partial_{\tilde{\theta}} \Psi\right)}{r r^{+} \sin \theta} .
$$




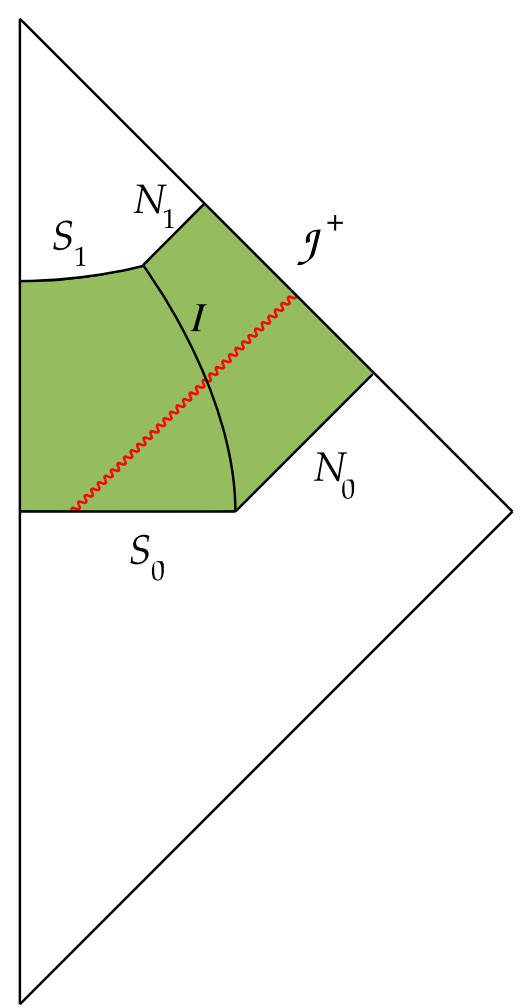

Figure 3. By combining a Cauchy and a characteristic scheme one solves for the entire spacetime of interest, including $\mathscr{I}^{+}$(future null infinity), where the radiation is unambiguously defined. Initial data is specified at $S_{0}$ and $N_{0}$ and the two schemes are connected by a time-like interface $I$. Although in principle one could use the asymptotically null approach up to null infinity, experiments with the wave equation in spherical symmetry suggest that the scheme is less effective than that which uses a time-like boundary at very large distances [27.

Note the lack of second partial derivatives $\partial_{u}^{2}$, reflecting the fact that the $u=$ const surface is characteristic.

\subsection{Axisymmetry and first order reductions}

We focus on axisymmetric solutions of the equations (3) and (13), as we do not expect additional difficulties to arise in the general 3-dimensional case. This implies $\partial_{\phi} \Phi=0$ and regularity demands that, across the axis $\theta=0$ and $\theta=\pi$, the scalar field is an even function of $\theta$. 
Since the discretization of second order systems tends to be more subtle than that of fully first order systems [28, 29, 30, 31, 32, we perform a reduction to first order and discretise the resulting system. In the Cauchy region we introduce the auxiliary variables $T=\partial_{t} \Psi, R=\partial_{r} \Psi$ and $\Theta=\partial_{\theta} \Psi$. The reduced system is

$$
\begin{aligned}
& \partial_{t} T=\frac{4 M}{r^{+}} \partial_{r} T+\frac{r^{-}}{r^{+}} \partial_{r} R-\frac{2 M}{r r^{+}} T+\frac{2 M}{r r^{+}} R-\frac{2 M}{r^{2} r^{+}} \Psi+\frac{\partial_{\theta}(\sin \theta \Theta)}{r r^{+} \sin \theta}(14) \\
& \partial_{t} R=\partial_{r} T, \\
& \partial_{t} \Theta=\partial_{\theta} T, \\
& \partial_{t} \Psi=T .
\end{aligned}
$$

We also have the constraint $\mathcal{C} \equiv \partial_{r} \Theta-\partial_{\theta} R=0$, which propagates with zero speed and therefore does not introduce complications at the boundary. This system is symmetrizable hyperbolic provided that $r>2 M$ [33]. It has non trivial characteristic speeds 1 and $-r^{-} / r^{+}$in the radial direction and the corresponding characteristic variables are

$$
w^{ \pm}=\frac{r^{+}}{r^{-}} T \pm R .
$$

To reduce equation (13) to first order we introduce $\tilde{R}=\partial_{\tilde{r}} \Psi, V=2 r / r^{-} \partial_{u} \Psi-\partial_{\tilde{r}} \Psi$ and $\tilde{\Theta}=\partial_{\tilde{\theta}} \Psi$. The reason for this choice of auxiliary variables is that $\partial_{\tilde{r}}$ and $2 r / r^{-} \partial_{u}-\partial_{\tilde{r}}$ are null vectors. We will refer to $\tilde{R}$ and $V$ as null variables. The reduced system is

$$
\begin{aligned}
\partial_{\tilde{r}} V & =-\frac{2 M}{r r^{-}} V-\frac{2 M}{r^{2} r^{-}} \Psi+\frac{\partial_{\tilde{\theta}}(\sin \theta \tilde{\Theta})}{r r^{-} \sin \theta}, \\
2 \partial_{u} \tilde{R} & =\frac{r^{-}}{r} \partial_{\tilde{r}} \tilde{R}+\frac{2 M}{r^{2}} \tilde{R}-\frac{2 M}{r^{3}} \Psi+\frac{\partial_{\tilde{\theta}}(\sin \theta \tilde{\Theta})}{r^{2} \sin \theta}, \\
2 \partial_{u} \tilde{\Theta} & =\frac{r^{-}}{r}\left(\partial_{\tilde{\theta}} V+\partial_{\tilde{\theta}} R\right), \\
2 \partial_{u} \Psi & =\frac{r^{-}}{r}(V+\tilde{R}) .
\end{aligned}
$$

To reach $\mathscr{I}^{+}$we compactify the characteristic radial coordinate $\tilde{r}$ by introducing a new independent variable $\xi$ such that

$$
\tilde{r}=r_{i}+\frac{2}{\pi}\left(\xi_{\infty}-\xi_{i}\right) \tan \left(\frac{\pi}{2} \frac{\xi-\xi_{i}}{\xi_{\infty}-\xi_{i}}\right) .
$$

In the last equation $r=r_{i}$ and $\xi=\xi_{i}$ represent the location of the interface boundary in the Cauchy and characteristic coordinate systems, respectively. The surface $\xi=\xi_{\infty}$ corresponds to future null infinity. Effectively, this amounts to replacing in system (19)- (22) the partial derivatives with respect to $\tilde{r}$ with

$$
\partial_{\tilde{r}}=\frac{\partial \xi}{\partial \tilde{r}} \partial_{\xi}=\cos ^{2}\left(\frac{\pi}{2} \frac{\xi-\xi_{i}}{\xi_{\infty}-\xi_{i}}\right) \partial_{\xi}
$$

There are coefficients in equation (19) that contain indetermined forms, which can be explicitly evaluated using the limit

$$
\lim _{\xi \rightarrow \xi_{\infty}} \tilde{r}^{2} \frac{\partial \xi}{\partial \tilde{r}}=\frac{4}{\pi^{2}}\left(\xi_{\infty}-\xi_{i}\right)^{2} .
$$

We typically use $r_{i}=\xi_{i}=10 M$ and $\xi_{\infty}=20 M$. 


\subsection{Interface treatment}

A necessary condition for well-posedness for the interior Cauchy problem is that at the boundary and at the interface data is prescribed to the incoming characteristic variables. If no data is specified to these variables, then one can expect to lose uniqueness. The characteristic region can provide accurate data for the incoming mode by setting

$$
\left.w^{+}\right|_{r=r_{i}}=\left.\tilde{R}\right|_{\xi=\xi_{i}} .
$$

On the other hand, the characteristic problem requires data for the outgoing null variable $V$. This is done by setting

$$
\left.V\right|_{\xi=\xi_{i}}=[T-R]_{r=r_{i}} .
$$

\subsection{Treatment of future null infinity}

Future null infinity corresponds to the boundary surface $\xi=\xi_{\infty}$. Here, as a result of the compactification, the speed of the incoming variable $\tilde{R}$ approaches zero, so there are no incoming modes (the surface is null). No data is required at this boundary and its numerical treatment is particularly simple. See subsection 3.5.

\section{Finite difference scheme}

Stable finite difference approximations of the initial-boundary value problem for the wave equation in the Cauchy region (not coupled to the characteristic problem) are available. For example, by approximating the spatial derivatives using finite difference operators that satisfy the summation by parts rule [34, it is possible to construct schemes which conserve a discrete energy 33. 35], a positive definite quadratic form of the dependent variables. Boundary conditions can then be imposed either using Olsson's method [36, 37, 38, or using penalty terms [39, 40, 41].

It is possible to obtain energy estimates in the characteristic region 42, 43, 44, and in turn for the coupled Cauchy-characteristic problem. Ideally, one would like to achieve similar estimates at the discrete level. Although we have been able to formally extend these estimates to the discrete case, the semi-discrete problem that we consider fails to admit solutions for generic initial data.

Formally, one can obtain discrete estimates for the semi-discrete system

$$
A^{u} \partial_{u} w+A^{x} D_{x} w+A^{y} D_{y} w+A^{z} D_{z} w=0
$$

where $w$, which is a shorthand for $w_{i j k}(u)$, is a vector-valued grid-function having the dynamical variables as components and the variables $x, y$ and $z$ are discretised with indices $i, j$ and $k$. Furthermore, $u$ is left continuous, $y$ and $z$ are periodic, the matrices are constant and symmetric and $A^{u}$ is singular. The operators $D_{x}, D_{y}$ and $D_{z}$ are discrete finite difference operators. The operators $D_{y}$ and $D_{z}$ are centered finite difference approximations of $\partial_{y}$ and $\partial_{z}$. The operator $D_{x}$ approximates $\partial_{x}$ and satisfies the summation by parts rule

$$
\sum_{i=0}^{N}\left(D_{x} w_{i}\right)^{T} v_{i} \sigma_{i} h+\sum_{i=0}^{N} w_{i}\left(D_{x} v_{i}\right) \sigma_{i} h=w_{N} v_{N}-w_{0} v_{0},
$$

$\S$ The summation by parts rule is the discrete analogue of the integration by parts rule: $\int_{a}^{b} w^{\prime}(x) v(x)+$ $w(x) v^{\prime}(x) d x=w(b) v(b)-w(a) v(a)$. 
where $\sigma_{i}$ are appropriate weights (explicit examples of weights and operators can be found in 34) and $h$ is the spatial mesh spacing. System (28) represents the semidiscrete approximation of (19)- 222). Without loss of generality we can assume that $A^{u}$ is diagonal and therefore at least one equation in system (28) does not contain derivatives with respect to $u$. Multiplying on the left by $w^{T}$ and using the fact that the matrices are constant and symmetric, we get

$$
\begin{aligned}
& \left(\partial_{u} w\right)^{T} A^{u} w+w^{T} A^{u} \partial_{u} w+\left(D_{x} w\right)^{T} A^{x} w+w^{T} A^{x} D_{x} w \\
& +\left(D_{y} w\right)^{T} A^{y} w+w^{T} A^{y} D_{y} w+\left(D_{z} w\right)^{T} A^{z} w+w^{T} A^{z} D_{z} w=0 .
\end{aligned}
$$

Summing over the indices corresponding to $x, y$ and $z$ and integrating in $u$ gives the equality

$$
\left[\sum_{i=0}^{N} w_{i}^{T} A^{u} w_{i} \sigma_{i} h\right]_{u=u_{0}}^{u=u_{1}}+\int_{u=u_{0}}^{u=u_{1}}\left[w_{i}^{T} A^{x} w_{i}\right]_{i=0}^{i=N} d u=0,
$$

where $[f(u)]_{u=u_{0}}^{u=u_{1}}=f\left(u_{1}\right)-f\left(u_{0}\right),\left[f_{i}\right]_{i=0}^{i=N}=f_{N}-f_{0}$, the index $i$ corresponds to the $x$ direction, and the indices corresponding to $y$ and $z$ are suppressed. Despite the estimate, a solution of (28) does not exist. This can be seen by looking at the equation that does not involve derivatives in the $u$ direction. The system $D_{x} w=F$, for generic $F$, is overdetermined because the operator $D_{x}$ is singular.

Since the summation by parts approach does not appear to work, our guiding principle will be to discretise the problem in a way consistent with the continuum problem\|, for which estimates do exist. Numerical experiments will be crucial for establishing convergence of the resulting scheme.

\subsection{Cauchy grid}

The discretization of the Cauchy problem is done in a standard way: the derivatives are approximated with centered fourth order accurate finite difference operators

$$
D_{0}\left(1-\frac{h^{2}}{6} D_{+} D_{-}\right) \text {, }
$$

where $D_{0} w_{i}=\left(w_{i+1}-w_{i-1}\right) /(2 h), D_{+} w_{i}=\left(w_{i+1}-w_{i}\right) / h$, and $D_{-} w_{i}=\left(w_{i}-w_{i-1}\right) / h$. When applied to a grid-function $w_{i}$, operator (31) gives

$$
\left(-w_{i+2}+8 w_{i+1}-8 w_{i-1}+w_{i-2}\right) /(12 h) .
$$

The boundaries, the interface and the axis of symmetry are implemented using ghost points. To eliminate the black hole singularity from the domain we use excision. At the boundary the ghost points are populated $\boldsymbol{\uparrow}$ using extrapolation and by overwriting the incoming characteristic fields, if any are present, at the boundary point. See Appendix B.2 of [32 and [45. Near the axis of symmetry these are populated using the regularity conditions, i.e., using the fact that $\Psi$ is an even function of $\theta$ across the axis. The interface boundary is treated as if it was an outer boundary, with the only exception that the incoming mode is provided by the characteristic evolution.

We also add some artificial dissipation to the scheme of the form $\sigma h^{5}\left(D_{+} D_{-}\right)^{3}$, explicitly

$$
\sigma\left(w_{i+3}-6 w_{i+2}+15 w_{i+1}-20 w_{i}+15 w_{i-1}-6 w_{i-2}+w_{i-3}\right) / h,
$$

\| A finite difference scheme is said to be consistent if solutions to the continuum problem satisfy the scheme to an order greater or equal to 1 in the mesh spacing.

I Populating ghost points means assigning the values of the dynamical variables at these points. 
with $\sigma=0.075$ [46] and use fourth order Runge-Kutta to integrate in time with a Courant factor of 0.5. Although we are investigating a linear problem, the partial differential equations have variable coefficients. A certain amount of artificial dissipation is desirable for stability and will most likely be necessary in the non-linear case.

\subsection{Characteristic grid}

The discretization of the evolution part of the system, Eqs. (20)-(22), is done as in the Cauchy case. Equation (19) treated as an ordinary differential equation in $\tilde{r}$, the last two terms in the right hand side taking the role of forcing terms. It is advanced in the $\tilde{r}$ direction by using fourth order Runge-Kutta (with a "time" step equal to the radial mesh spacing). This requires the evaluation of the coefficients and forcing terms of (19) at intermediate time steps. The missing data is generated with fourth order interpolation

$$
U_{i+1 / 2}=\left(-U_{i-1}+9 U_{i}+9 U_{i+1}-U_{i+2}\right) / 16 .
$$

Note that to start the integration we need the fields at the grid-point $i=-1$. We use fourth order extrapolation to populate this point.

The integration of $V$ along the $\tilde{r}$ direction is done at each intermediate time step of the global time integrator. Artificial dissipation is added to the evolution in the $u$ direction.

\subsection{Axis of symmetry}

We use ghost points to treat the axis $\theta=0, \pi$. The dynamical variables $\Psi, T, R$, $\tilde{R}$ and $V$ are even functions of $\theta$ along the axis, and $\Theta$ and $\tilde{\Theta}$ are odd functions. In particular, we impose that $\Theta=\tilde{\Theta}=0$ on the axis.

\subsection{Interface treatment}

Data is communicated at the interface at each intermediate time step: the Cauchy code requires the incoming variable $w^{+}$, Eq. (26), and the characteristic code needs the outgoing null variable $V$, Eq. (27). The Cauchy and characteristic grids are touching, rather than overlapping. This avoids complications with causality which would arise if the $u=$ const. slice intersected the domain of dependence of the $t=$ const slice.

At the interface boundary we implement condition (26) by solving for $T$ and $R$ the system of equations

$$
\begin{aligned}
& \frac{r^{+}}{r^{-}} T+R=\tilde{R}, \\
& \frac{r^{+}}{r^{-}} T-R=\frac{r^{+}}{r^{-}} T_{\text {old }}-R_{\text {old }} .
\end{aligned}
$$

\subsection{Treatment of future null infinity}

The only difficulty at the outer boundary of the characteristic grid is that some coefficients contain indetermined forms " $0 \cdot \infty$ ". We use the limit (25) to evaluate these. We populate the ghost points by extrapolating all fields. As there are no incoming modes no further action is taken. 


\subsection{The algorithm}

In this subsection we list the basic steps of our algorithm.

(i) Give initial data to all fields in the Cauchy grid ( $\Psi$ and $T$ can be given arbitrarily, but $R$ and $\Theta$ have to obey their definition constraints). Give initial data to all fields on the characteristic grid ( $\Psi$ can be given arbitrarily, $\tilde{R}$ and $\tilde{\Theta}$ have to satisfy their definition constraints), with the exception of the null variable $V$.

(ii) Impose regularity on the axis, impose interface boundary conditions (26) and (27). Integrate Eq. (19) using fourth order Runge-Kutta (use fourth order Lagrange interpolation to populate the coefficients and forcing terms of the differential equation). Extrapolate all fields in the radial direction to populate the ghost points. Impose regularity again.

(iii) Evaluate the right hand sides of the evolution equations (because we use ghost points this can be done at every interior, and boundary/axis/interface point) and calculate the solution at the next intermediate time step.

(iv) Repeat from point (ii) until a full fourth order Runge-Kutta time step is taken.

(v) Repeat from point (iil) and take as many Runge-Kutta time steps are needed to reach the final time.

\section{Numerical tests}

\subsection{Convergence test}

To test for convergence we set $M=0$, construct an exact solution by translating the spherically symmetric solution $\Phi=f(t-r) / r$ along the axis by an amount $\delta$,

$$
\Psi=r \Phi=\frac{r}{\hat{r}} f(t-\hat{r}),
$$

where $\hat{r}^{2}=r^{2}-2 r \delta \cos \theta+\delta^{2}$. The expressions for $T, R, \Theta$ and $\tilde{R}, V, \tilde{\Theta}$ can be readily computed. We monitor how the $L_{2}$-norm of the error between the numerical and exact solution,

$$
\left\|w-u_{\text {(exact) }}\right\|=\left(\sum_{j}\left(w_{j}-u_{(\text {exact })}\right)^{2} h_{r} h_{\theta}\right)^{1 / 2}
$$

scales with resolution. Since the exact solution is known, we only need to use two resolutions (coarse and fine) to test for convergence. In the expression above $h_{r}$ and $h_{\theta}$ represent the mesh spacing and the sum is extended to all grid-points of the Cauchy and charateristic grids.

The coarse resolution is $200 \times 200$ in each grid. The fine resolution is obtained by doubling the number of grid-points in each direction. The Cauchy slices extend from $r=2$, where an artificial boundary is introduced to avoid the $r=0$ coordinate singularity, to $r=10$, the location of the spherical interface boundary. We use a time step $\Delta t=\Delta u=0.02$ for the coarse case and half it for the fine case. The test confirms that the scheme is indeed fourth order convergent. See figure 4 


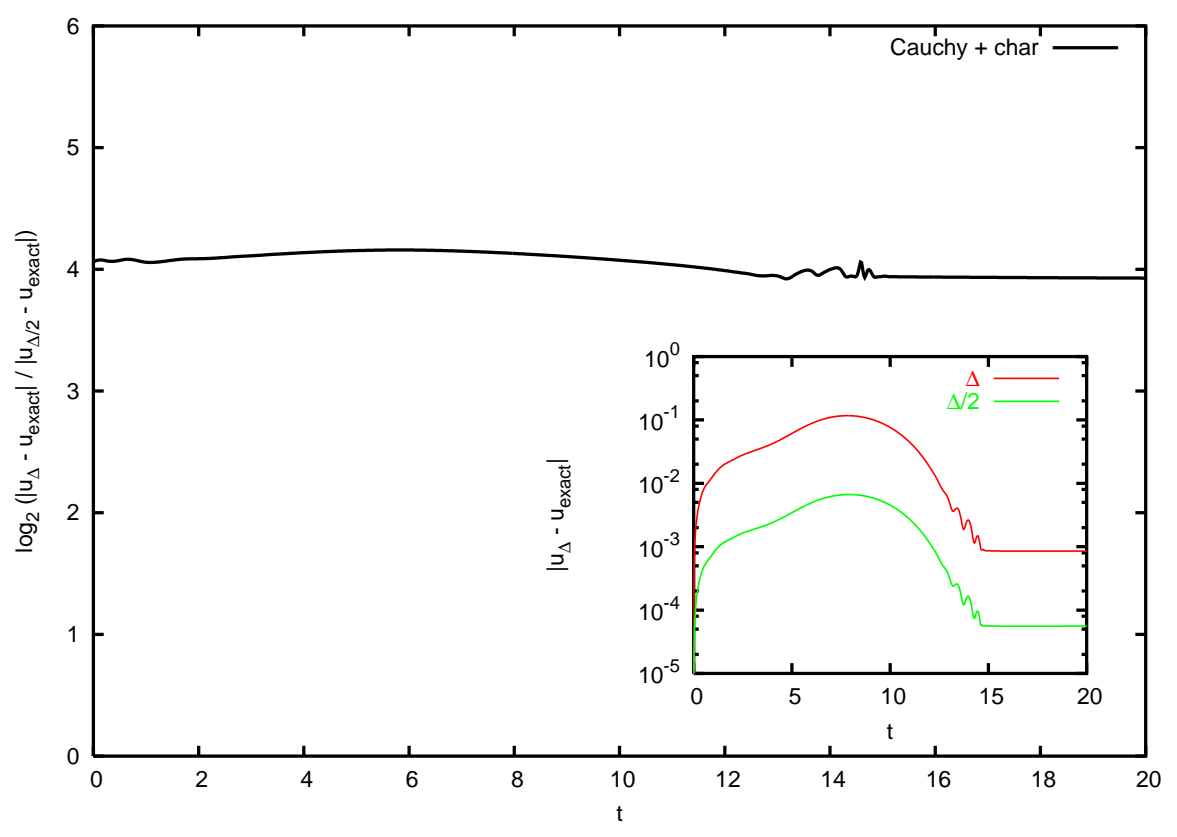

Figure 4. This plot illustrates the experimental convergence rate of the scheme as a function of time. The initial pulse, which is of compact support, completely leaves the domain shortly before $t=15$. The numerical errors left in the computational domain converge away at the correct rate. The inset shows the $L_{2}$-norms of the errors at the different resolutions.

\subsection{Tail decay}

Having established that the scheme is fourth order accurate we want to determine whether it can accurately produce the late time tail decays [47, 48, 49. The field $\Psi$ is expected to decay as $t^{-3}$ (along a time-like curve and assuming spherically symmetric initial data), because of the scattering of the field off the curvature of spacetime asymptotically far from the black hole. We set $M=1$, give initial data to $\Psi$ consisting of a spherically symmetric pulse with compact support,

$$
\Psi(0, r, \theta)= \begin{cases}\cos ^{6}(r-4), & |r-4| \leq \pi / 2, \\ 0, & \text { otherwise, }\end{cases}
$$

and set its time derivative equal to zero. We use $r_{i}=\xi_{i}=10$ and $\xi_{\infty}=20$. We monitor the value of $\Psi$ at three different locations: $r=6, r=16(\xi=15)$ and $\xi=\xi_{\infty}$ (i.e., at $\mathscr{I}^{+}$). Using 400 grid-points in the radial direction in both grids and very few in the angular direction, we obtain good agreement with the expected result. See figure 5 The errors in the angular direction are of round-off order, i.e., $10^{-15}$ times smaller than the solution.

\section{Conclusion}

To study gravitational radiation at future null infinity, where it is unambiguously defined, numerical schemes have to evolve the fields up to that surface. An approach which would allow for this is Cauchy-characteristic matching (CCM), whereby 


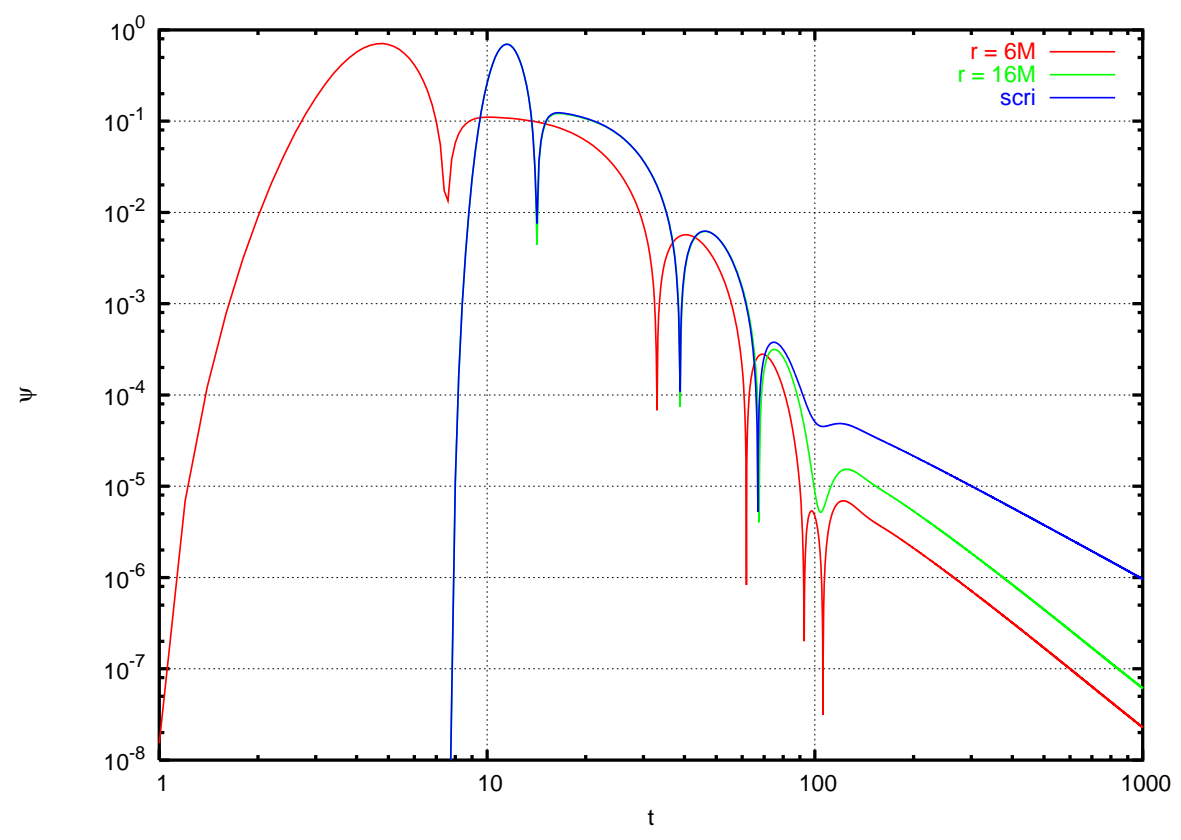

Figure 5. Amplitude of the scalar field as measured by observers at fixed radial coordinate $r=6 M, r=16 M$ and at $\mathscr{I}^{+}$. Whereas the first observer is in the Cauchy region, the other two are in the characteristic region. Note that the decay rate measured by the observer at infinity differs from that of the observers at a finite distance. At $t=800 M$ the ratio $d(\ln \Psi) / d(\ln t)$ is -2.92 , for the $r=6 M$ and $r=16 M$ observers, and -1.97 , for the observer at $\mathscr{I}^{+}$.

Cauchy and characteristic evolutions are matched across a time-like world-tube. This approach presents many advantages over other boundary conditions, such as maximally dissipative or absorbing boundary conditions. CCM makes it possible, for example, to compute accurate waveform and polarisation properties at infinity. It is a highly computationally efficient technique which can eliminate the undesirable reflections and inaccuracies that other boundary conditions would introduce [13.

In this work we described a fourth order accurate algorithm for a Cauchycharacteristic formulation of the wave equation written in first order form. By compactifying the radial coordinate in the characteristic region we can evolve all the way to $\mathscr{I}^{+}$. We carried out convergence tests showing that the scheme is fourth order convergent and were able to reproduce the expected tail decay rate. The interface was implemented using touching grids, which share a common smooth boundary, and by communicating the relevant characteristic/null variables.

The success for the scalar field case is clearly only a necessary condition for the fully nonlinear case. The nonlinear extension of this work will require a careful choice of coordinate conditions and attention to the propagation of constraints [50, 51, 52, 53]. It will be crucial to establish how constraint violations propagate across the domain, so that the interface treatment can be done consistently. In three dimensions one would still require a smooth interface boundary implemented through either overlapping grids [46] [54, 55], which work well with simultaneous slices and can also allow for moving boundaries, or touching grids 56, 57, 58. 


\section{References}

[1] Pretorius F 2005 Phys. Rev. Lett. 95121101

[2] Baker J, Centrella J, Choi D, Koppitz M and van Meter J 2006 Phys. Rev. Lett. 96111102

[3] Campanelli M, Lousto C, Marronetti P and Zlochower Y 2006 Phys. Rev. Lett. 96111101

[4] Allen E, Buckmiller E, Burko L and Price R 2004 Phys. Rev. D 70044038

[5] Dafermos M and Rodnianski I 2004 Preprint gr-qc/0403034

[6] Frauendiener J 2004 Living Rev. Relativity 7 1. URL (cited on 7 April 2006) http://www.livingreviews.org/lrr-2004-1

[7] Kansagra A 2003 "Coordinate compactifications and hyperboloidal slices in numerical relativity" LIGO/Caltech undergraduate project, unpublished. For an online abstract, see http://www.ligo.caltech.edu/LIGO_web/students/ugprojects03.html

[8] Misner C and Scheel M 2003 "Wave propagation with hyperboloidal slicings", talk given 12 June 2003 at KITP, unpublished. For an online version see http://online.kitp.ucsb.edu/online/gravity03/misner

[9] Misner C 2004 Preprint gr-qc/0409073

[10] van Meter J, Fiske D and Misner C 2006 Preprint gr-qc/0603034

[11] Sachs R 1962 Proc. R. Soc. London, Ser. A 270 103-126

[12] Bondi H, van der Burg M and Metzner A 1962 Proc. R. Soc. London, Ser. A 269 21-52

[13] Winicour J 2001 Living Rev. Relativity 4 3. URL (cited on 7 April 2006) http://www.livingreviews.org/lrr-2001-3

[14] d'Inverno R 1992 Approaches to Numerical Relativity (Cambridge University Press, Cambridge)

[15] Clarke C and d'Inverno R 1994 Class. Quantum Grav. 11 (6) 1463-1448

[16] Clarke C, d'Inverno R and Vickers J 1995 Phys. Rev. D 52 (12) 6863-6867

[17] Dubal M, d'Inverno R and Clarke C 1995 Phys. Rev. D 52 (12) 6868-6881

[18] d'Inverno R and Vickers J 1996 Phys. Rev. D 54 (8) 4919-4928

[19] d'Inverno R and Vickers J 1997 Phys. Rev. D 56 (2) 772-784

[20] Dubal M, d'Inverno R and Vickers J 1998 Phys. Rev. D 58044019

[21] d'Inverno R, Dubal M and Sarkies E 2000 Class. Quantum Grav. 17 (16) 3157-3170

[22] Sperhake U, Sjödin K and Vickers J 2001 Phys. Rev. D 63024011

[23] Sperhake U, Sjödin K and Vickers J 2001 Phys. Rev. D 63024012

[24] Bishop N, Gómez R, Holvorcem P, Matzner R, Papadopoulos P and Winicour J 1996 Phys. Rev. Lett. 76 (23) 4303-4306

[25] Bishop N, Gómez R, Holvorcem P, Matzner R, Papadopoulos P and Winicour J 1997 J. Comput. Phys. 136 (1) 140-167

[26] Gómez R, Laguna P, Papadopoulos P and Winicour J 1996 Phys. Rev. D 54 (8) 4719-4727

[27] Calabrese G, Gundlach C and Hilditch D 2006 Class. Quantum Grav. 23 4829-4845

[28] Szilagyi B, Kreiss H-O and Winicour J 2005 Phys. Rev. D 71104035

[29] Motamed M, Babiuc M, Szilagyi B, Kreiss H-O and Winicour J 2006 Phys. Rev. D 73124008

[30] Calabrese G 2005 Phys. Rev. D 71 027501(4)

[31] Calabrese G, Hinder I and Husa S 2005 Preprint gr-qc/0503056

[32] Calabrese G and Gundlach C 2006 Class. Quantum Grav. 23 S343-S367

[33] Calabrese G, Lehner L, Neilsen D, Pullin J, Reula O, Sarbach O and Tiglio M 2003 Class. Quantum Grav. 20 L245-L252

[34] Strand B 1994 J. Comput. Phys. 11047

[35] Calabrese G, Lehner L, Reula O, Sarbach O, and Tiglio M 2004 Class. Quantum Grav. 21 5735-5758

[36] Olsson P 1995 Math. Comp. 641035

[37] Olsson P 1995 Math. Comp. 64 S23

[38] Olsson P 1995 Math. Comp. 641473

[39] Carpenter M, Gottlieb D and Abarbanel S 1994 J. Comput. Phys. 111

[40] Carpenter M, Nordstrom J and Gottlieb D 1999 J. Comput. Phys. 1482341

[41] Nordstrom J and Carpenter M 2001 J. Comput. Phys. 173

[42] Frittelli S 2004 J. Phys. A: Math. Gen. 37 8639-8655

[43] Frittelli S 2005 J. Phys. A: Math. Gen. 38 4209-4221

[44] Frittelli S 2005 Phys. Rev. D 71024021

[45] Gustafsson B, Kreiss H-O and Oliger J 1995 Time dependent problems and difference methods (New York: Wiley)

[46] Calabrese G and Neilsen D 2005 Phys. Rev. D 71 124027(20)

[47] Price R 1972 Phys. Rev. D 52419

[48] Gundlach C, Price R and Pullin J 1994 Phys. Rev. D 49883 
[49] Barack L 1999 Phys. Rev. D 59044017

[50] Calabrese G, Lehner L and Tiglio M 2002 Phys. Rev. D 65 104031(13)

[51] Calabrese G, Pullin J, Reula O, Sarbach O and Tiglio M 2003 Commun. Math. Phys. 240 377-395

[52] Szilagyi B and Winicour J 2002 Phys. Rev. D 66064019

[53] Kreiss H-O and Winicour J 2006 Class. Quantum Grav. 23 S405-S420

[54] Thornburg J 2004 Class. Quantum Grav. 213665

[55] Calabrese G and Neilsen D 2004 Phys. Rev. D 69 044020(21)

[56] Lehner L, Reula O and Tiglio M 2005 Class. Quantum Grav. 22 5283-5322

[57] Schnetter E, Diener P, Dorband E and Tiglio M 2006 Class. Quantum Grav. 23 S553-S578

[58] Diener P, Dorband E, Schnetter E and Tiglio M 2005 Preprint gr-qc/0512001 